\title{
COVID-19 and academic publishing ranking
}

Constantinus Politis

MD, DDS, MM, MHA, PhD

Professor \& Chairperson

Editor-in-Chief

Dear Readers,

In a section of Foreign Affairs (Volume 100, Number 5, 2021), Dr. Chonira Aturupane of Stanford University points out that during the COVID-19 pandemic a number of challenges stood in the way of effective policymaking: the prevalence of unreliable information; the degree to which uncertainty and unpredictable factors can derail the best-laid plans; the importance of comprehensive and feasible implementation plans. We will concentrate on the first issue, the importance of credible data.

Credible data, reliable information in science does not equal the absence of fake news. Credibility in scientific reporting requires at least the following dimensions to be assessed: method and data transparency; adequate sample size and power; validity of the data; adequate use of secondary data; analytic reproducibility and robustness; effect replicability; independent study sponsoring. Respecting all these requirements should lead to evidence-based data and evidence-based medicine (EBM).

Yet, on the reporting site in journals, the cornerstone of EBM has shifted far too much from original research towards the higher positioned systematic reviews and meta-analysis. Many thousands of pages have been filled with answering questions nobody was waiting for. Further on, unexperienced readers should not assume that level 1 evidence is always the best choice or appropriate for the research question. An item often overlooked is the natural history of a condition. For reviewers of most journals it is nearly impossible to adequately assess all items which define the quality of a study: randomization, blinding, a description of the randomization and blinding process, description of the number of subjects who withdrew or dropped out of the study; the confidence intervals around study estimates, and a description of the power analysis and the statistical methods used. Most reviewers are no statisticians.

This only can be solved if the number of journals is sufficiently reduced to allow for a thorough change of the review process. Before getting to the clinical reviewers, language experts should filter out inadequate manuscripts and trained statisticians should filter out studies which are underpowered, unvalidated or illdesigned. Only then should clinical reviewers assess the manuscript. This requires journals to be digital only with a well-developed staffing connected in the cloud and funded by independent academic consortia. The digital only version, and of course open access, allows for post-hoc comments or corrections to be added as a section to published papers.

As for systematic reviews and meta-analysis, these are very often conducted by PhD students or Master students in the fullfillment of a PhD or a Masters Program. It is cheap, fills pages, whether the question is relevant or not. It is very often easily accepted in high ranked journals, creating easily gained impact factors, citations and h-index points, and all of this usually at a young research age.

It is time to attach less importance to systematic reviews if the question they try to answer is not relevant. More is to be gained in well-conducted clinical research, but this is tedious work requiring resources and an experienced research team. In the clinical reality of surgical departments this is a rare phenomenon. The consequence of this surgical reality is that younger surgeons write manuscripts and publish articles and seasoned surgeons write book chapters or books or invited sessions in journals.

There is yet another way skilled surgeons can contribute to journals and science. Through their scientific societies, often in joined efforts with representatives of sister organisations, they can aim for concensus 
statements and guidelines. Professor Hendrik Terheyden, editor-in-chief of the International Journal of Implant Dentistry has guided the International Academy for Oral and Facial Rehabilitation (IAOFR) to work along these lines. The IAOFR, presided by Prof. Dr. Piet Haers, is an international group with more than 30 years of continuous history in the field of oral and facial rehabilitation of patients with innate or acquired tooth loss or defects of the jaws and the craniofacial region. This group has an international composition, high scientific expertise and independence. Consensus Statements and Guidelines can target a worldwide audience of practitioners, clinicians and patients. The methodology used is in accordance with the GIN-McMaster Guideline Development Checklist, the AWMF Guidance Manual and Rules for Guideline Development, and the policies of the Guidelines International Network. All this information is freely accessible on the internet. Consensus Statements and Guidelines allow for a corridor of accepted clinical treatment or pathways, excluding under-therapy, over-therapy, false therapy, obsolete therapy. Consensus Statements and Guidelines try to identify a standard of care and have the character of a recommendation to clinicians in standard clinical situations and a standard clinical environment without any legal force. Consequently, clinicians can deviate from recommendations in specific cases and situations. Consensus Statements and Guidelines can also be of help for informed patients for participative therapeutic decisions. Guidelines also provide tools for policymakers.

Conflicts of interest need to be addressed in a transparent way. The policy of an independent guideline group requires a declaration, evaluation and management of conflicts of interest of every participant of the guideline group because the guideline should not be vulnerable to competing interests of industry companies or competing societies afterwards. A listing of possible conflicts of interest of the participants should be forwarded to all participants in advance of the consensus meeting.

Since medicine is a quickly evolving field each statement and guideline expires 3 years after publication. The 'Terheyden-Haers doctrine' not only allows seasoned and skilled surgeons to combine surgical experience with common sense and scientific rigor but to translate the surgeon's group-knowledge into answers to questions which are relevant to patients and society at large.

Journals will remain necessary for those who pursue academic careers and are in need of citations, $\mathrm{h}$-index scores to advance in the academic ranking. The perverse side-effect however is that Universities and Academic Institutions in so-doing make publishers of scientific journals with paid 'open access' filthy rich, while at the same time depriving a mass of interested parties of scientific information. It is high time that universities embrace unpaid open access and look for alternatives beyond the $h$-indexes and citation indices to appreciate the scientific value of young academics.

Sincerely yours,

Constantinus Politis $[$ -

MD, DDS, MM, MHA, PhD

Professor \& Chairperson

Editor-in-Chief 